POS PROCEEDINGS

\title{
Measurement of the tau lepton reconstruction and identification performance in the ATLAS experiment using pp collisions at $\sqrt{s}=13 \mathrm{TeV}$
}

\author{
Michael Hübner*, on behalf of the ATLAS Collaboration \\ University of Bonn, Germany \\ E-mail: michael.huebnerecern.ch
}

\begin{abstract}
Tau lepton decays into hadrons are important for many physics analyses performed at the LHC. To achieve high precision in the measurements, the performance of the reconstruction and identification algorithms used for hadronically decaying tau leptons have to be well-known. This article details the performance measurements at ATLAS for data collected at the LHC in the years 2015 and 2016. The discussed algorithms, i.e. the trigger efficiency, the identification of hadronic tau decays, and the energy calibration, are determined to a level of precision of a few percent.
\end{abstract}

Sixth Annual Conference on Large Hadron Collider Physics (LHCP2018)

4-9 June 2018

Bologna, Italy

\footnotetext{
* Speaker.
} 


\section{Introduction}

In this article the three main algorithms concerning the tau lepton reconstruction and identification in the ATLAS experiment [1] and their performance measured in $p p$ collisions delivered by the LHC will be introduced. The performance of these algorithms is of great importance to a variety of physics analyses involving hadronically decaying tau leptons, like the Higgs boson $(\mathrm{H})$ $H \rightarrow \tau \tau$ coupling analysis or beyond the Standard Model (BSM) searches.

\section{Reconstruction, identification, and triggering}

The main trigger used for example by the $H \rightarrow \tau \tau$ coupling analysis is designed to select events with two hadronically decaying tau leptons $\left(\tau_{\text {had,vis }}\right)$. This trigger uses an online identification algorithm, i.e. this algorithm is applied before the data is permanently stored. The goal of this identification is to keep as many events with real tau leptons as possible while rejecting as many events with quark- and/or gluon-initiated jets as possible, since the production cross section for these jets is much larger than the one for real tau leptons. To achieve a good separation between tau leptons and jets, variables are used that are for example sensitive to the shower shape in the calorimeters [2].

A similar identification algorithm is applied in the offline reconstruction of the recorded events. The algorithm is based on a Boosted Decision Tree using input variables that are built upon information from the tracking and calorimeter systems. Three different working points are defined loose, medium,tight which yield efficiencies for real taus of $0.60(0.50), 0.55(0.40)$ and $0.45(0.30)$ for tau leptons with 1(3) track(s) after reconstruction and identification. The inverse background efficiency versus the signal efficiency is shown in Fig. 1(a).

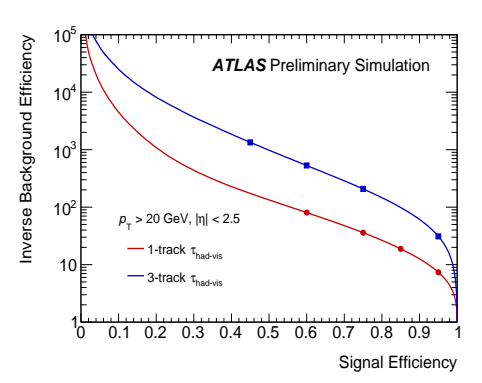

(a) RoC curve of the offline identification algorithm showing the inverse background efficiency against the efficiency of identifying a real $\tau_{\text {had,vis }}[5]$.

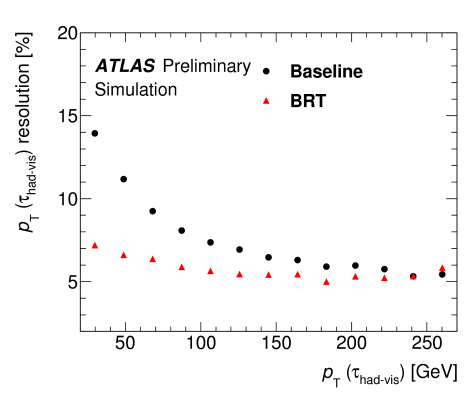

(b) Comparison of the $p_{\mathrm{T}}$ resolution using the new reconstruction and Boosted Regression Trees with the previous default tau lepton reconstruction [3].

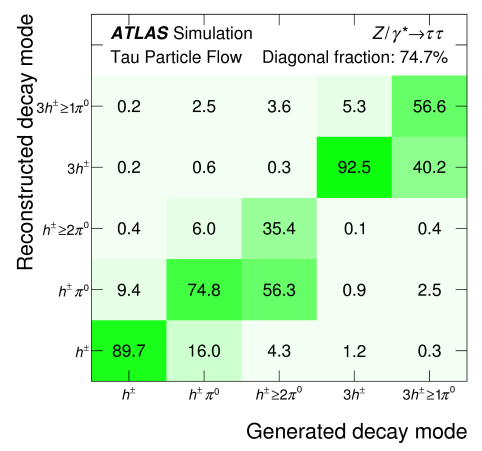

(c) Decay mode classification matrix for hadronically decaying tau leptons using the new reconstruction algorithm based on particle flow principles [4].

Figure 1: Features of the new tau lepton reconstruction used in the ATLAS experiment.

A new $\tau_{\text {had,vis }}$ reconstruction is available that is based on particle flow principles [4]. The reconstruction is still seeded by a jet clustering algorithm. However, in the subsequent steps, not 


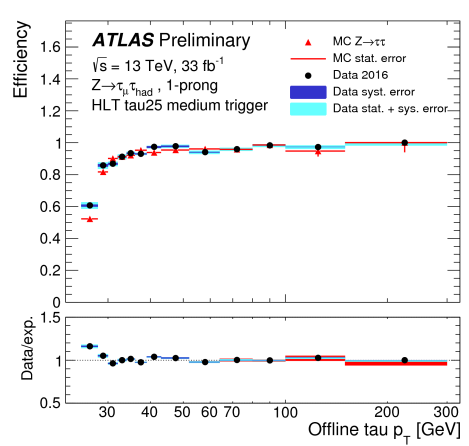

(a) Comparison of data and simulation of the trigger efficiency as a function of the tau $p_{\mathrm{T}}[2]$.

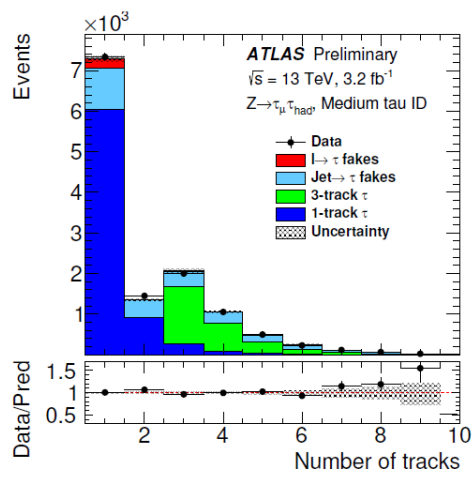

(b) Post-fit distribution of the $n_{\text {tracks }}$ variable used to derive the identification scale factors [3].

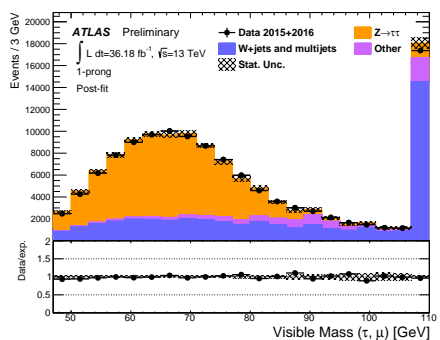

(c) Post-fit distribution of the visible mass of the two tau system used to derive the tau energy scale factors [5].

Figure 2: Distributions used to derive the scale factors and uncertainties for the different algorithms.

only the calorimeter information is used, but the tau lepton decay products, mainly charged and neutral pions, are reconstructed using the tracking system and the electromagnetic calorimeter, respectively. This improves the angular resolution compared to a calorimeter-only measurement by a factor of roughly two and the $p_{\mathrm{T}}$ resolution as shown in Fig. 1(b) [4]. To achieve this improvement in the $p_{\mathrm{T}}$ resolution, the $\tau_{\text {had,vis }}$ energy scale is calculated based on Boosted Regression Trees taking information provided by the new reconstruction into account, e.g. the hadronic tau decay mode. This decay mode classification is a new feature of the reconstruction algorithm where a diagonal efficiency of roughly $75 \%$ in simulation was achieved, see Fig. 1(c) [4].

\section{Performance Measurements}

The performance of the discussed algorithms is investigated using data collected by the ATLAS experiment in the years 2015 and 2016. These measurements provide correction factors to scale factors derived from simulation and systematic uncertainties on those.

The performance of all algorithms is measured using the $Z \rightarrow \tau_{\text {had }} \tau_{\mu}$ process since it can be selected with high purity and provides tau leptons in an important $p_{\mathrm{T}}$ range, for example for the $H \rightarrow \tau \tau$ coupling measurement. The event selection is similar for the different performance measurements. Events are selected where one of the two tau leptons, originating from the $Z$ boson decay, decays into a muon which can be tagged, i.e. the measurements trigger on the muon since it leaves a clear signature in the detector. The other tau lepton decays hadronically, mostly into a varying number of charged and/or neutral pions. The algorithms are probed on this hadronically decaying tau lepton. For QCD-induced multijet background and for W+jets background, datadriven techniques are used [3]. Similar approaches are used to extend the $p_{\mathrm{T}}$ range of the trigger efficiency measurement using $t \bar{t}$ processes. Figure 2 shows distributions of the observables and their background composition. 


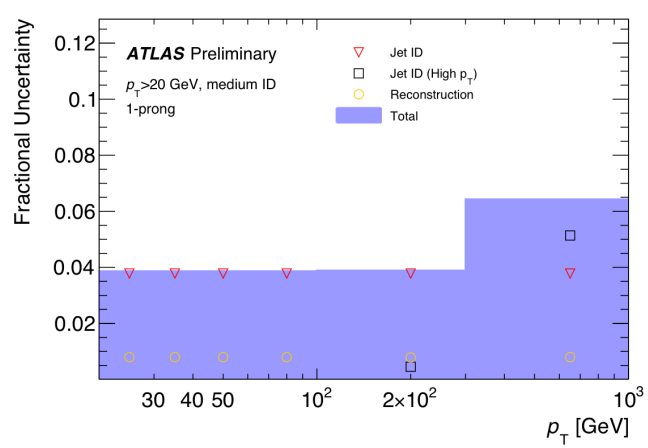

(a) Systematic uncertainties on the tau lepton identification for the medium working point and 1 -prong decays.

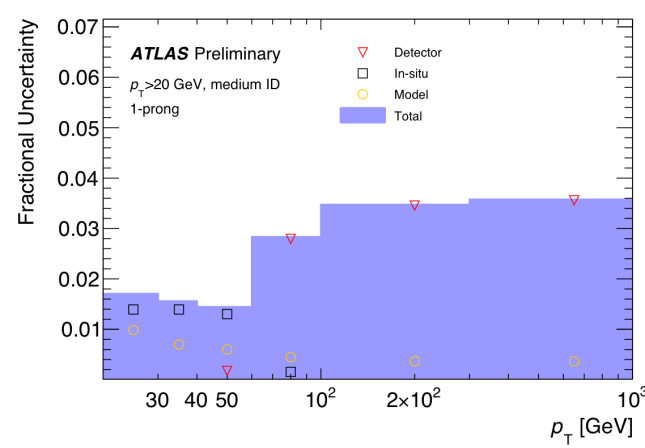

(b) Systematic uncertainties on the tau lepton energy scale for the medium working point and 1prong decays.

Figure 3: Systematic uncertainties for the tau lepton identification and the tau energy scale [5].

Correction factors for simulation are extracted by comparing simulation with data. Scale factors for the tau trigger efficiency are computed from the ratio of the efficiencies measured in simulation and data as a function of tau $p_{\mathrm{T}}$, see Fig. 2(a). The identification scale factors are derived from the ratio of efficiencies of data and simulation estimated from a fit to an observable that counts the number of tracks, $n_{\text {tracks }}$, associated to a tau candidate. This observable provides good separation between real and misidentified taus, as shown in Fig. 2(b). For the tau energy scale, a $\chi^{2}$ for varying energy scale values is minimized using the invariant visible mass of the two tau leptons, see Fig. 2(c). The scale factors are measured as a function of $p_{\mathrm{T}}$ and $\eta$ and are mostly consistent with unity. The systematic uncertainties on the identification and energy scale factors are summarized in Fig. 3. They are slightly rising for high tau $p_{\mathrm{T}}$ and are of the order of a few percent.

\section{Conclusions}

The performance of the tau lepton reconstruction and of the tau lepton identification algorithms used in the ATLAS experiment was discussed. Using data delivered by the LHC in the years 2015 and 2016, in similar analysis strategies for the different measurements, the uncertainties of the individual scale factors are of the order of a few percent while the scale factors themselves are mostly consistent with unity.

\section{References}

[1] ATLAS Collaboration, 2008 JINST 3 S08003.

[2] ATLAS Collaboration, ATLAS-CONF-2017-061, https://cds.cern.ch/record/2274201.

[3] ATLAS Collaboration, ATLAS-CONF-2017-029, https://cds.cern.ch/record/2261772.

[4] ATLAS Collaboration, Eur. Phys. J. C 76, no. 5, 295 (2016) [arXiv:1512.05955 [hep-ex]].

[5] ATLAS Collaboration, https://twiki.cern.ch/twiki/bin/view/AtlasPublic/TauPublicResults. 